\title{
LA FRAYE DU SANDRE (Lucioperca lucioperca L.) DANS L'ÉTANG DE SANGUINET
}

\author{
par M. LAURENT, C. GARAICOECHEA \\ Station d'Hydrobiologie I.N.R.A. - B.P. 79 - 64200 BIARRITZ \\ et J.-M. COUDIN \\ Section de plongée du B.E.C. (Bordeaux Etudiants Club)
}

Depuis une dizaine d'années environ, le Sandre a èté introduit dans les étangs landais et en particulier dans l'étang de Cazaux-Sanguinet. Les pêcheurs avaient reconnu l'existence des frayeres de Sandre sur l'herbier de Caton (herbier d'Elodea crispa), à une profondeur moyenne de $1,10 \mathrm{~m}$, ce qui concordait avec les conditions de frai classiquement admises (HUET, 1970).

En mars-avril 1971 et 1972 des plongeurs archéologues prospectant des vestiges gallo-romains, constitués de fragments de poteries et de tuiles disséminés parmi des rocailles, récemment découverts (TOBIE et al., 1972) sur le fond du lac de Sanguinet, ont été l'objet de la part des Sandres d'attaques allant jusqu'à des morsures. Ces derniers faits ont èté rapportés par CAPDEVIELLE (1972).

Lors d'une plongée effectuée sur le site, le 12 avril 1973 (température de l'eau en surface : $12 \circ \mathrm{C}$ ) les auteurs ont constaté la présence d'œufs de poissons sur des rocailles et vestiges gallo-romains, à $6 \mathrm{~m}$ de fond: ils ont èté attaqués par des Sandres de $50 \mathrm{~cm}$ de long environ qui ont mordillé l'extrémité d'un bâtonnet en bois de $15 \mathrm{cr}$ qu quils tenaient à la main.

En ce qui concerne les conditions de ponte du Sandre, si SCHAPERCLAUS (1962) admet la possibilite de ponte sur des rocailles, il semble que dans la littérature (HUET, 1970; SCHAPERCLAUS, 1962; ARRIGNON, 1970) les profondeurs signalées ne dépassent guère $1 \mathrm{~m}$ alors qu'à Sanguinet il s'agit, rappelons-le, de fonds de 6 mètres. 
Des comportements d'agressivité des poissons après la ponte ont ete observès en particulier chez l'épinoche par TINBERGEN (1953): de même ARRIGNON (1970) signale qu'en pisciculture des Sandres mordent une main touchant un nid artificiel; ce comportement est semblable à celui observé à Sanguinet lors de la collecte des rocailles, fragments de poterie et tuiles supportant le frai. A notre connaissance, l'agressivitè du Sandre vis-à-vis de l'homme en milieu naturel n'avait pas été signalée et il est curieux de constater que ce comportement arrive à perturber l'examen de sites archéologiques sous-lacustres.

\section{REFERENCES BIBLIOGRAPHIQUES}

ARRIGNON 1., 1970. Aménagement piscicole des eaux intérieures. 1 vol., 643 p., SEDETEC Ed., Paris

CAPDEVIELLE P., 1972. Quelques considèrations nouvelles sur le Sandre. Bull. Inf. Cons. Sup. Pêche, 8, $7-9$.

HUET M., 1970. Traité de pisciculture. 4e édition, 1 vol., 718 p., Ch. de Wyngaert Ed., Bruxelles.

SCHAPERCLAUS W., 1962. Traité de pisciculture en étang. 1 vol., 620 p. Vigot frères Ed., Paris.

TINBERGEN N., L'étude de l'instinct. 1 vol., 308 p., Payot Ed., Paris.

TOBIE J.-L., RICHIR C., COUDIN J.-M., COLOMBO J.-F., 1972. Vestiges galloromains sous le lac de Sanguinet. Archeologia, 48, 33-37. 